\title{
The Means to an End: Phonology's Prominence in a Reading Task
}

by Anna Grabovac

\section{Introduction}

The degree of influence of the phonology versus the visual cues of printed text during the reading process has been a subject of linguistic research for several decades. The debate primarily centers around whether the phonology or the visually-perceived, lexical spelling of a word has a greater effect on how the reader interprets the word. Depending upon which property takes precedence in the decoding process, it is possible to misinterpret the meaning of an individual word.

Studies have been conducted in an attempt to explain the cognitive steps involved in the reading process. In "The Functions of Phonology in the Acquisition of Reading: Lexical and Sentence Processing," Johnston et al. (1995) describe two experiments that examine the influence of phonology on reading-for-meaning tasks. Based on the collective results of Experiments 1 and 2, the authors argue that rather than the use of direct lexical access when making judgments about the meaning of sentences, certain circumstances prompt children (their subjects) to refer to phonology before activating the lexical meaning of an individual word. However, in the case of homophonic pairs, similar phonology activates a spelling-check procedure that decreases a greater number of errors in judgment than the sole use of phonology. Thus, the results of this study indicate that children employ both phonology and knowledge of spelling during the reading process.

In "A ROWS is a ROSE: Spelling, Sound, and Reading," Van Orden (1987) reaches a slightly different conclusion. He describes three experiments that reflect the impact of "stimulus word phonology" on the performance of college students in lexical identification tasks. Overall, his primary argument seems to be that while phonology may act as an early constraint in word identification, a combination of phonology and spelling is necessary for correct identification. Furthermore, phonology may play a role in covariant learning, which can accomplish direct access. Whereas the conclusions of Johnston et al. may be regarded as supporting the dual-route model of reading, Van Orden argues that the reader may employ phonology merely as an aid for direct access.

\subsection{Hypothesis}

Though designed differently, Van Orden's 1987 study initially inspired the inclusion of both homophone foils and spelling foils in my experiment. Thus, I formulated my hypothesis based on the results of his experiments. 
However, Johnston et al. discovered that certain circumstances prompt children to refer to phonology before activating the lexical meaning of a word, so one goal of my study was to determine whether or not a highpressure situation could be labeled as one of these "circumstances." I tested this by forcing participants to make a split-second decision regarding the correctness of sentences. Although Van Orden reasoned that both phonology and spelling are required for correct identification, the Johnston et al. study prompted me to conjecture that phonology may take precedence in a high-pressure situation. Therefore, based on the results of these previous studies, the hypothesis at the outset of my study was as follows: If a reader is forced to provide split-second ratings of correctness for pairs of printed sentences and audio recordings that contain either a homophone-usage error or the misspelling of a target word, the reader is more likely to give a false positive rating for the sentences containing homophones because the easy access of phonology supersedes the visual processing of words.

\section{Methods}

\subsection{Subjects}

The subjects consisted of a group of 20 participants who were all undergraduate students at Boston College. All of the participants were native speakers of English. They were recruited through personal outreach (e.g. email, word-of-mouth). In order to protect individual privacy, each participant was assigned an identification code from P1-P20.

\subsection{Materials}

This experiment entailed the use of Microsoft PowerPoint on a laptop. Twenty sentences, which were created by the author, were individually displayed for a fixed 3-4 seconds (depending upon the length of the sentence) on the computer screen. The sentences (though presented randomly) were divided into four groups: Group I-correct English utterances, Group II-nonsense sentences, Group III-homophone foils, and Group IV-spelling foils. The foils in Group III were chosen in relation to a logical homophone, while those in Group IV were formed by reversing the order of two adjacent letters. For example, consider the following: ${ }^{1}$

- $\quad$ The dog chased the mailman. (correct)

- $\quad$ Planet rocks screams fishing greenery. (nonsense)

- Shouts of acclimation accompanied his speech. (homophone foil)

- She records her thouhgts. (spelling foil)

\footnotetext{
${ }^{1}$ For a complete list of sentences in the order in which they were used in this experiment, see Appendix 1.
} 
No two sentences of the same category occurred immediately in sequence. As each sentence appeared on the screen, an audio recording of the sentence accompanied the text. The recordings were provided by an outside party who did not otherwise participate in the experiment. The sentences containing homophone and spelling foils were articulated as if they contained no errors. Together with the nonsense utterances, the audio recordings served as distracters from the less obvious homophone and spelling foils.

Participants were provided with a pen and a sheet on which to record their judgments of the correctness of each sentence. At the top of the sheet were the following instructions: Decide if each sentence presented is either correct or incorrect in English. Circle YES for correct and NO for incorrect. The sheet was divided into two sections: Practice and Task. The Practice section was numbered 1-3, while the Task section was numbered 1-20, each number corresponding to a sentence, with $Y E S$ and $N O$ listed next to each number. ${ }^{2}$

\title{
2.3. Procedure
}

This experiment required subjects to participate in an evaluation of sentence correctness. Before the start of the experiment, the participant was seated in front of the computer, which displayed a blank, orange screen. Then, the participant received the response sheet along with a pen. He or she heard the following instructions:

\begin{abstract}
This study investigates the correspondence between phonology, or the sounds of words, and reading. A series of 20 sentences will individually appear on the screen. As each sentence appears, it will be read aloud by an audio recording. Your job is to decide whether or not each sentence is correct or incorrect in English. Circle YES on the sheet for correct and NO for incorrect. We will first practice with three example sentences, and then I will prompt you when the real task begins.
\end{abstract}

I individually presented the three practice sentences and then provided the desired answer for each one. They were as follows (with the correct responses):

\section{1) The knight fought the dragon. YES}

2) Her work exeeded all expectations. NO

3) Clocks sings shoe are shy. NO

Note that Sentence 2 was intended to inform the participant that correct spelling should factor into his or her judgment of correctness, without exposing the use of homophones in the actual task.

After the instructions were given and the practice section was completed, the participant carried on with the true task. As each sentence/audio pair appeared for 3-4 seconds, the participant had this time to mark his or her decision before the appearance of the next sentence. The goal was to

\footnotetext{
${ }^{2}$ See Appendix 2.
} 
preclude the participant from having enough time to debate the correctness of each sentence. The participant's split-second inclination was most desirable in order to best analyze the influence of phonology on reading.

Including the initial instructions and practice sentences, the total task took about 10 minutes per participant. On several occasions, it was also administered to multiple participants at once, provided that they all had unobstructed access to both the audio and the screen. Once I had completed the entire task, I collected each participant's response sheet and translated the results into two graphs. The first graph depicts the number of YES and NO responses for each sentence, while the second graph shows the number of erroneous responses for each sentence. See Figures 1 and 2, respectively.

Figure 1

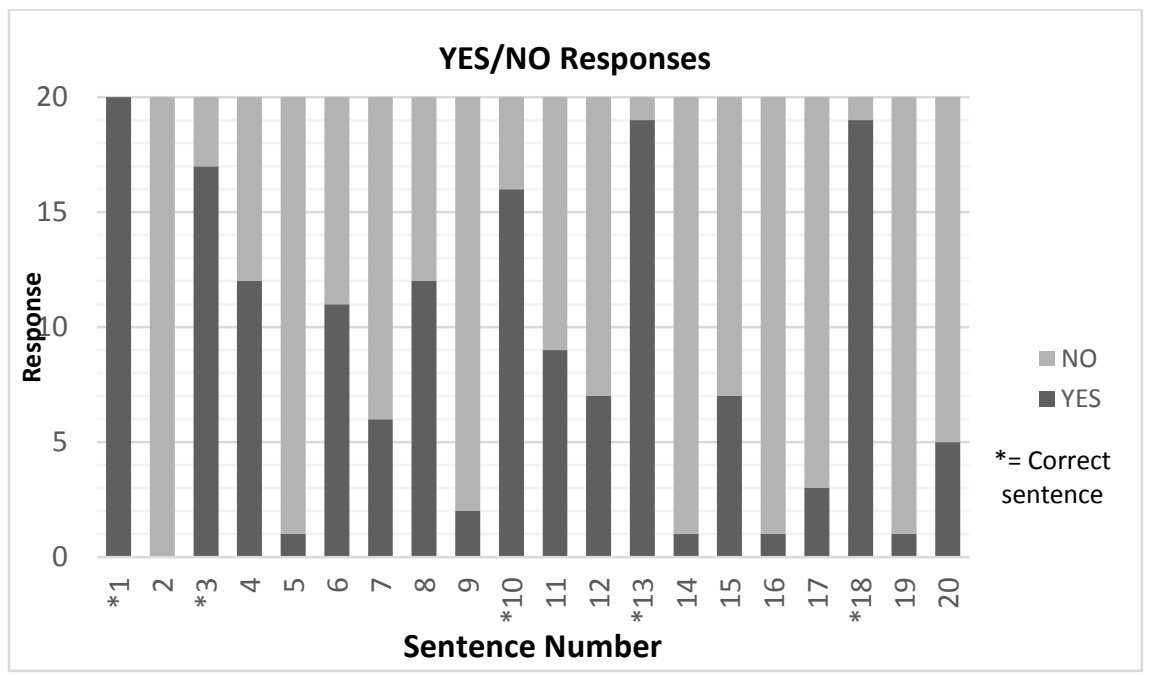

Figure 2 


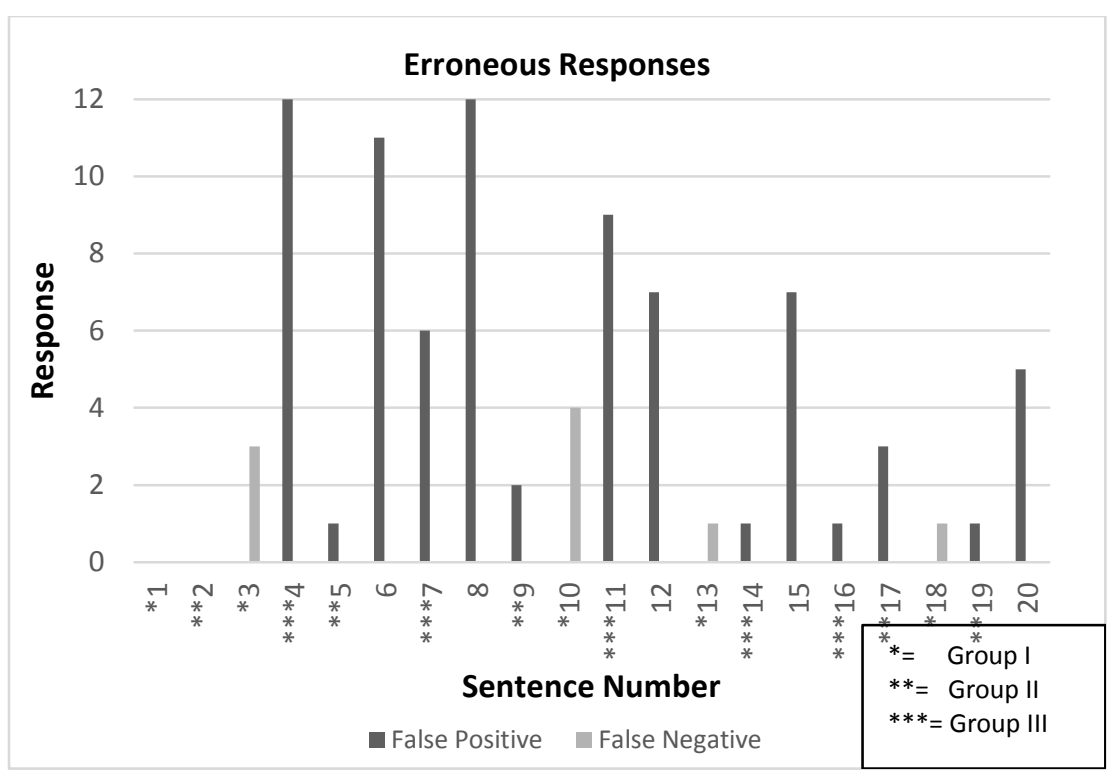

\section{Results}

The results of the correctness identification task only partially reflect the predictions set forth in the hypothesis. The graph in Figure 1 shows that the first two statements caused no confusion for participants. However, Statement 3 begins a trend of debate over the correctness of statements that continues for the remainder of the task. Figure 2 illustrates that participants made few errors in the identification of sentences belonging to Group I. Group II generally expresses the same trend. Group III, on the other hand, generates many false positive ratings, as reflected in Figure 2. However, it appears that near the end of the task, many of the participants began to catch the presence of the homophone foils, as the graph shows a drastic decrease in the number of false positives. Group IV also has a high number of false positive ratings - nearly across the board. Contrary to the predictions set forth in my hypothesis, the spelling foils in Group IV were more often erroneously identified than were the homophone foils.

\section{Discussion}

The results of this study confirm the hypothesis to the extent that the participants seem to rely heavily on phonology when forced to make a quick decision regarding the correctness of a written statement. This is evident in the large number of false positive ratings across both Group III and Group IV statements. Though less obvious, this observation may also be extended to Group I and Group II statements, as statements in these categories "sound" either standard or blatantly unacceptable. Because the participants did not have enough time to thoroughly read and evaluate each sentence, one may infer that they immediately resorted to phonological assessment. 


\subsection{Group I Analysis}

In order to fully digest the data produced from the task, it is constructive to individually examine each of the four groups of sentences, beginning with Group I. Overall, this group has very few false negative ratings. However, it is surprising that there are any false negative ratings at all. Group I sentences should have been obviously correct, but only Statement 1 has zero false negative ratings. Statements 3,13 , and 18 also have relatively low false negative ratings, but Statement 10, I eat the orange, has four erroneous ratings. Perhaps these results can be attributed to the location of the statement within the collection. It appears to be a general trend that the participants began to notice the presence of homophone and spelling foils as the task progressed, so they may have falsely anticipated a ruse in this statement. Furthermore, the use of the present simple tense in conjunction with a definite article sounds somewhat unnatural.

\subsection{Group II Analysis}

Because the statements in Group II were designed to be obvious nonsense utterances, the false positive responses are somewhat unexpected. The third practice sentence should have conveyed the idea that statements of this type are to be regarded as incorrect. Furthermore, Statement 2 has no erroneous responses, so every participant began the experiment with the desired view. The most surprising result is Statement 17, which has the highest number of false positive ratings-three. The statement, Planet rocks screams fishing greenery, does not "sound" remotely correct in terms of English morphology. One possible explanation is that participants understood planet rocks as a single constituent, perhaps as the name of a location. Only in this sense would planet rocks agree with screams in terms of number. Another plausible justification is that the statement occurs subsequent to Statement 15, a spelling foil with a high number of false positive ratings. Comparable to the phenomenon observed among Group I statements, the participants may have simply succumbed to habit and anticipated the positive rating.

\subsection{Combined Analysis of Groups III \& IV}

Group III sentences contain homophone foils. At the outset of the experiment, it seemed reasonable that the homophone foils would be the most difficult for participants to correctly identify, given the results of Johnston et al. and Van Orden. Thus, Group III should have had the highest number of false positive ratings, according to the hypothesis. However, the results of this experiment undeniably show that the number of false positive ratings across Group III is second to that of Group IV.

Although the results do not exactly reflect the findings of previous studies, they may be reconciled upon further reflection. It appears that participants did rely heavily on the phonological properties of each sentence throughout 
the experiment. However, the results of the Van Orden study suggest that phonology is a means to direct access. Thus, given the speed at which the sentences were presented, it is not surprising that participants frequently missed the spelling foils as they resorted to phonological assessment. The spelling foils were also pronounced in the audio as if they contained no errors, so they essentially functioned the same as the homophone foils. Often, the spelling of the homophone foils looks more obviously incorrectespecially when there is a change in the number of letters-such as the substitution of inns for ins in Statement 16, or faze for phase in Statement 7. The spelling foils, on the other hand, were each formed by switching the order of two adjacent letters, so they may be less conspicuous.

Let us now examine the homophone foils more closely by referring back to Figure 2. Sentence 4 appears to have confused the majority of participants, as it has twelve false positive ratings. Sentence 7 has six false positive ratings, while Statement 11 has nine, and Statements 14 and 16 each have one. Upon closer analysis, these results are consistent with the spelling observations discussed above. The homophone in Statement 4, acclimation, only contains one letter different from acclamation. Statement 11 is similar in that borders lacks only the $a$ of boarders. In Statements 7, 14, and 16, the homophone is more obvious. The use of faze in 7 is noticeable since it lacks three of the letters of phase. Additionally, please in 14 is a fairly common word, which may have made it more easily recognizable for the participants. The use of inns in 16 is relatively conspicuous-again due to a change in the number of letters.

As previously mentioned, Group IV has the highest number of erroneous ratings of any group. Statement 6 has eleven false positives, 8 has twelve, 12 and 15 have seven, while 20 has five. Let us consider Statements 6 and 8 , which have the most false ratings with thouhgts and sunhsine, respectively. A possible explanation for their high number of ratings is their location toward the onset of the experiment. After Statement 8, the remaining spelling foils decrease in the number of false positive ratings as participants began to figure out the essence of the task. Overall, it is apparent that the original hypothesis is incorrect in its prediction of the homophone foils containing more false positive ratings than the spelling foils. However, the existence of false positive ratings in both the homophone and spelling foils does reflect the participants' initial reliance on phonology, rather than immediate lexical access. The general decrease in erroneous ratings with the experiment's progression provides possible evidence for the dual-route model of reading, in which both phonology and lexical access play a role.

\section{Conclusion}

While the results of this experiment suggest that participants may initially resort to evaluating words based on phonology in a high-pressure situation, the fact that most participants began to figure out the gist of the task must not be overlooked. This phenomenon may be attributed to the frequency and general appearance of the foils-factors that should be more carefully considered if an experiment similar to this one were to take place. As 
participants started to figure out the task, it is logical to infer that they had to access the lexicon. Furthermore, they had to either ignore or look beyond the phonology presented. Thus, comparable to the results of Johnston et al., one may conclude that phonology and spelling are used in conjunction. Although the dual-route model seems plausible, the participants may have used phonology as a means to direct access, as Van Orden suggests. The results of this study do not indisputably confirm either model. Therefore, further research is necessary in order for more definite conclusions to be drawn. This same experiment could be carried out without the accompanying audio recording. In this case, it would be easier to determine whether or not participants initially resort to the use of phonology. If they do, one would expect more false positive ratings across the homophone foils and fewer across the spelling foils. These results could be evaluated alongside the results of the original study, and perhaps a more explicit conclusion could be made regarding the steps in the reading process.

\section{Apparatus}

\section{BIBLIOGRAPHY}

Johnston, R. S., Thompson, G. B., Fletcher-Flinn, C. M., \& Holligan, C. (1995). The Functions of Phonology in the Acquisition of Reading: Lexical and Sentence Processing. Memory \& Cognition, 23 (6), 74966.

Van Orden, G.C. (1987). A ROWS is a ROSE: Spelling, Sound, and Reading. Memory \& Cognition, 15 (3), 181-98.

\section{APPENDIX 1}

\section{Sentences}

Practice

1. The knight fought the dragon. $\mathbf{C}$

2. Her work exeeded all expectations. $\mathbf{S}$

3. Clocks sings shoe are shy. I

Task

1. The dog chased the mailman. $\mathbf{C}$

\begin{tabular}{|l|}
\hline Key \\
C-Correct/Group I \\
I-Incorrect/Group II \\
H-Homophone Foil/Group III \\
s-Spelling Foil/Group IV \\
\hline
\end{tabular}

2. Swimming red yellows is went. I

3. He catches the ball. C

4. Shouts of acclimation accompanied his speech. $\mathbf{H}$

5. Spicy ate maps climbed. I 
6. She records her thouhgts. $\mathbf{S}$

7. The moon is in the crescent faze. $\mathbf{H}$

8. The vacationers bask in the sunhsine. $\mathbf{S}$

9. Cat orange mountains bark. I

10. I eat the orange. $\mathbf{C}$

11. The borders paid their monthly rent. $\mathbf{H}$

12. He attempted a complicated manuver. $\mathbf{S}$

13. She read the letter. $\mathbf{C}$

14. The king heard the please of the people. $\mathbf{H}$

15. The colunms fortify the building. $\mathbf{S}$

16. He knows the inns and outs of baseball. $\mathbf{H}$

17. Planet rocks screams fishing greenery. I

18. The cat watches the mouse. C

19. Purples the dishwasher the sun. I

20. The mistakes were not ovbious at first. $\mathbf{S}$

\section{APPENDIX 2}

Decide if each sentence presented is either correct or incorrect in English. Circle YES

for correct and NO for incorrect.

\section{A. Practice}

1. YES NO

2. YES NO

3. YES NO

B. Task

1. YES NO

2. YES NO

3. YES NO

4. YES NO

5. YES NO

6. YES NO

7. YES NO

8. YES NO

9. YES NO 
10. YES NO

11. YES NO

12. YES NO

13. YES NO

14. YES NO

15. YES NO

16. YES NO

17. YES NO

18. YES NO

19. YES NO

20. YES NO 